\title{
Cryopreservation of immature Parkia speciosa Hassk. zygotic embryonic axes following desiccation or exposure to vitrification solution.
}

\begin{abstract}
This work reports on the cryopreservation of immature zygotic embryonic axes (EA) of petai (Parkia speciosa Hassk.) for the first time. Two cryopreservation protocols, namely desiccation and vitrification method were tested individually using excised EA. Desiccation of EA to lower moisture content (MC) reduced the survival percentage but a drastic decline in survival percentage $(\sim 20 \%)$ was recorded at $16 \%$ MC prior to exposure to $\mathrm{LN}$, rendering the EA to be sensitive to desiccation. Cryopreservation of EA after desiccation, irrespective of the MC, did not result in any survival. On the other hand, post-cryopreservation survival was obtained when the EA were exposed to plant vitrification solution-2 (PVS2) for 75-105 min. The best results were obtained when the EA were exposed to PVS2 for 90 min with an average recovery of $55.5 \%$. EA recovery into whole plantlets was obtained when the EA were cultured on MS medium supplemented with 2 gl-1 activated charcoal and $0.1 \mathrm{mgl}-1$ of the plant growth regulators $\alpha$-naphthalene acetic acid, 6-benzylaminopurine and gibberellin A3, each. EA, exposed for less than 75 min and more than 105 min to PVS2, did not show any survival after cryopreservation. The optimization of exposure time is necessary to increase survival. This study has shown that the employment of suitable method is important for conservation using cryopreservation.
\end{abstract}

Keyword: Desiccation; Liquid nitrogen; PVS2; Recalcitrant seeds; Survival; Tree; Vitrification. 